\title{
PREVALÊNCIA EPIDEMIOLÓGICA DE INTERNACÕES POR TRANSTORNO MENTAL DEVIDO AO USO DE ÁLCOOL NA MICRORREGIÃO DE ARAÇUAÍ, SITUADA NO VALE JEQUITINHONHA, MINAS GERAIS, BRASIL
}

\section{EPIDEMIOLOGICAL PREVALENCE OF HOSPITALIZATIONS FOR MENTAL DISORDER DUE TO THE USE OF ALCOHOL IN THE MICRO-REGION OF ARAÇUAÍ, LOCATED IN THE JEQUITINHONHA VALLEY, MINAS GERAIS,} BRAZIL

\author{
Dayane Alves Barreto dos Santos \\ Psicóloga, graduada na UNIPAC TO, \\ Pós Graduada em Saúde Mental, Brasil \\ dayanebarretopsi@gmail.com
}

\section{Resumo}

A atuação da Psicologia em políticas públicas tem papel fundamental na Atenção, Prevenção e Promoção da saúde biopsicossocial, integrando-se como uma ferramenta no desafio ao que se refere transtorno mental devido ao uso de álcool. O objetivo deste trabalho é investigar a prevalência de transtornos mentais devido ao uso de álcool no Vale Jequitinhonha, especificamente na microrregião de Araçuaí, Minas Gerais, Brasil. A microrregião de Araçuaí fica no Médio Jequitinhonha e abrangem os municípios de Araçuaí, Caraí, Coronel Murta, Itinga, Novo Cruzeiro, Padre Paraíso, Ponto dos Volantes e Virgem da Lapa. Os dados utilizados foram do SIH/SUS no período de 2008 a 2014. Num contexto mais amplo, vinculam-se pesquisas bibliográficas sobre os transtornos mentais e comportamentais, assim se preocupa em compreender através das leituras que se têm feito dos problemas relacionados ao alcoolismo. A pesquisa foi realizada no SIH/SUS no período de 2008 a 2014, com um total de 44 internações. O município de Araçuaí teve maior ocorrência de internações, porém Virgem da Lapa teve a maior média em relação à população. No período indicado houve um crescimento de internações, as variáveis que tiveram maior prevalência foi: homens, sexo masculino, idades entre 30 a 49 anos e pessoas na cor parda. Ao concluir este estudo, verificamos a necessidade de pesquisas que apontem o uso de álcool no Vale Jequitinhonha e a atuação das técnicas de psicologia na prevenção do problema. 
Palavras- chave: Saúde Mental; Transtorno mental; Álcool; Sistema Único de Saúde.

\section{Abstract}

The operation of Psychology in public policies has an important role in the attention; prevention and promotion of biopsychosocial health, integrating itself as a tool when it comes to the mental disorders due to alcohol consumption. The aim of this paper is to investigate the prevalence of mental disorders due to alcohol consumption in Vale Jequitinhonha, specifically in the small region of Araçuai, Minas Gerais, Brazil. The small region of Araçuai is located in the Médio Jequitinhonha and it covers the counties of Carai, Coronel Murta, Itinga, Novo Cruzeiro, Padre Paraíso, Ponto dos Volantes e Virgem da Lapa. The data used are sourced in the SIH/SUS in the period from 2008 to 2014. In a broader context, it entails bibliographic researches about mental and behavioral disorders, thus it intends to understand it through the approaches that have been done about the problems related to alcoholism. The research was done in the SIH/SUS in the period from 2008 to 2014 , with a total of 44 hospitalisations. The county of Araçuai had the biggest occurrence of hospitalisations, however, Virgem da Lapa had the biggest average in terms of population. In the mentioned period there was a rise in hospitalisations, the tags that had the biggest prevalence were: men; male gender; aged 30 to 49 and people of mixed skin. In the conclusion of this paper, we confirmed the necessity of researches that point out the use of alcohol in the Vale Jequitinhonha and also the need for the application of the techniques of Psychology in the prevention of the problem.

Keywords : Mental health; Mental disorder; Alcohol; Single Health System 


\section{Introdução}

Os transtornos mentais e comportamentais são classificados pela Classificação Internacional de Doenças (CID 10) (1993) e o Manual Diagnóstico e Estatístico de Transtornos Mentais (DSM IV) (1995), como: transtornos de ansiedade; transtorno de pânico; transtorno depressivo; transtorno bipolar; fobias; transtorno de somatização; transtorno de aprendizagem; transtornos psicóticos; transtorno obsessivo compulsivo; transtorno de ajustamento; mania e ciclotimia; transtorno devido ao uso de drogas e outros.

A delimitação do tema desta pesquisa foi questionar qual o índice de internação por transtorno mental devido o uso de álcool, elaborou-se um trabalho por meio de dados obtidos através do Sistema de Internações Hospitalares do Sistema Único de Saúde (SIH/SUS) no período de 2008 a 2014, discutiu-se a relação existente entre transtornos mentais e comportamentais e o uso de álcool, em pacientes internados no Sistema Único de Saúde (SUS), especificamente na microrregião de Araçuaí, no Vale Jequitinhonha/MG.

Pressupõe que há um aumento de internações por este problema na região, isso seria devido "municípios com menor contingente populacional apresentarem o maior número de internações por alcoolismo" (MACHADO RM, COSTA JR ML. 2012, p78).

Nessa medida, este estudo objetivou mostrar por meio de alguns indicadores de Saúde disponíveis nos sistema de informação do Sistema Único de Saúde, a situação do uso de álcool na microrregião de Araçuaí, investigar sobre internações por transtorno mental devido ao uso de álcool, descrever aspectos epidemiológicos, caracterizar fatores de risco e favorecer que através dos resultados obtidos sobre a situação do alcoolismo, uma atuação da psicologia com a prevenção do uso de álcool.

${ }^{1}$ A Universidade Federal de Minas Gerais -UFMG diz que o Vale Jequitinhonha localiza-se no nordeste de Minas Gerais, banhado pelo Rio

\footnotetext{
${ }^{1}$ https://www2.ufmg.br/polojequitinhonha/O-Vale/Sobre-o-Vale
} 
Jequitinhonha, o Vale do Jequitinhonha ocupa uma área de $79 \mathrm{mil} \mathrm{km} 2$, com uma população de aproximadamente 980 mil habitantes onde mais de dois terços dela vive na zona rural. É composto, hoje, por 75 municípios, dos quais 52 estão organizados nas microrregiões Alto, Médio e Baixo Jequitinhonha, e 23 estão integrados à antiga área mineira da SUDENE e mais de dois terços dela vive na zona rural. Vários diagnósticos convergem em assinalar que as restrições hídricas e as secas periódicas são fatores cruciais para o baixo desempenho da agropecuária.

A microrregião de Araçuaí fica no Médio Jequitinhonha e abrangem os municípios de Araçuaí, Caraí, Coronel Murta, Itinga, Novo Cruzeiro, Padre Paraíso, Ponto dos Volantes e Virgem da Lapa.

Dadas às descrições demográficas supracitadas, foi feito levantamentos estatísticos referentes a transtornos mentais devido ao uso de álcool da região mencionada, através do conhecimento dos dados colhidos no banco de dados do DATASUS.

\section{Metodologia}

Trata-se de um estudo epidemiológico descritivo, cujos dados foram obtidos por meio de consulta na bases de dados disponibilizados pelo Departamento de Informática do Sistema Único de Saúde (DATASUS), no endereço eletrônico (http://www.datasus.gov.br), que foi acessado entre os meses agosto a outubro do ano de 2015.

A população do estudo foram pacientes que tiveram internação pelo SUS registrados pelo Sistema de Informações Hospitalares (SIH/SUS), residentes nos municípios da microrregião de Araçuaí, no período de 2008 a 2014, sendo que a microrregião compreende os municípios de Araçuaí, Caraí, Coronel Murta, Itinga, Novo Cruzeiro, Padre Paraíso, Ponto dos Volantes e Virgem da Lapa.

Esta pesquisa levantou dados com variáveis dependentes: transtornos mentais e comportamentais e independentes: Internações de pacientes por Transtorno Mental devido ao uso de álcool; Gênero, Faixa etária, Cor / Raça.

Os dados foram obtidos através do SIH/SUS (disponível no DATASUS), analisadas as informações referentes à prevalência de internações pelo SUS por transtornos mentais devido ao uso de álcool, por faixa etária, por gênero e por cor/raça, referentes aos municípios da microrregião de Araçuaí.

Os dados pesquisados foram entre os anos de 2008 a 2014, porém nas tabelas e gráficos estão a partir do segundo semestre de 2009, isso pode ser devido à ausência de lançamentos nos anos de 2008 ao primeiro semestre de 2009. 
As cidades de Coronel Murta, Itinga e Ponto dos Volantes não aparecem na pesquisa em geral, provavelmente não houve internações com a variável pesquisada no período de tempo em questão.

Finalmente foram analisados os dados do SIH/SUS, apresentando os índices de prevalência em gráficos, sendo que a análise estatística foi realizada através do software Excel@, onde os dados foram tabulados, analisados e apresentados através de gráficos. 


\section{Resultados e Discussão}

Resultado da pesquisa SIH/SUS na microrregião de Araçuaí, Vale Jequitinhonha, Minas Gerais, Brasil

A microrregião de Araçuaí fica no Médio Jequitinhonha e abrangem os municípios de Araçuaí, Caraí, Coronel Murta, Itinga, Novo Cruzeiro, Padre Paraíso, Ponto dos Volantes e Virgem da Lapa.

Os dados pesquisados foram entre os anos de 2008 a 2014, porém nas tabelas e gráficos estão a partir do segundo semestre de 2009, isso pode ser devido à ausência de lançamentos nos anos de 2008 ao primeiro semestre de 2009.

As cidades de Coronel Murta, Itinga e Ponto dos Volantes não aparecem na pesquisa em geral, provavelmente não houve internações com a variável pesquisada no período de tempo em questão.

De acordo com os dados coletados no SIH/SUS, no período de 2009 a 2014 foram internados 44 pacientes com diagnóstico de transtorno mental devido ao uso de álcool (Gráfico 1 )

Em ambos os municípios, como mostra o GRÁF. 1, houve variação com o número de internações.

No município de Araçuaí houve maior prevalência de internação por transtorno mental devido ao uso de álcool e o município de Caraí foi menor.

GRÁFICO 1- Internação por ano/mês de atendimento segundo o município por transtorno mental devido ao uso de álcool. 
Revista Multidisciplinar do Nordeste Mineiro, v1. 2020/01 ISSN 2178-6925

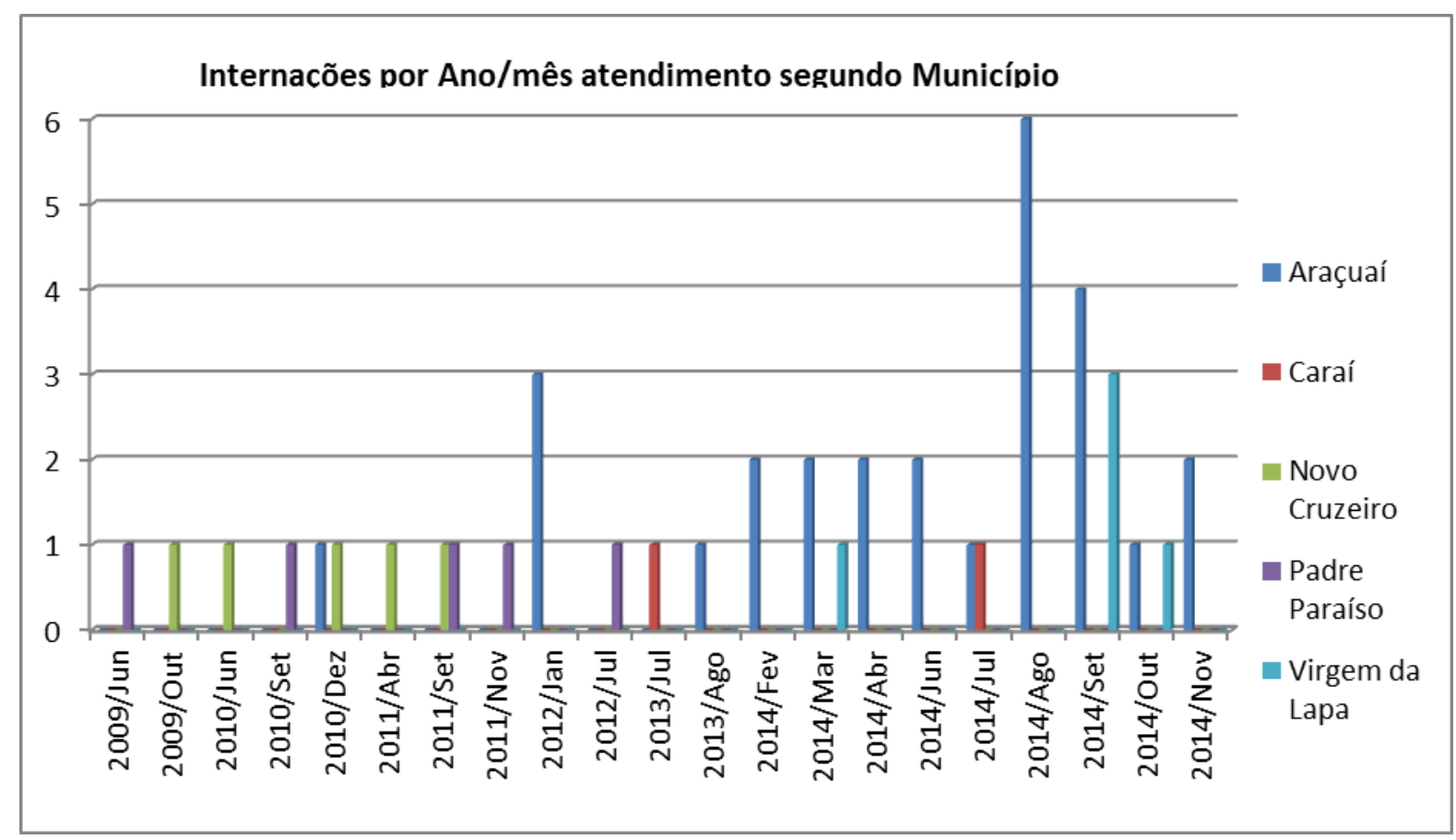

Fonte: SIH/SUS (2015)

Percebemos que o mês de agosto teve maior ocorrência de internações. GRÁFICO 2 - Média de internação por ano/mês de atendimento segundo o município por transtorno mental devido ao uso de álcool.

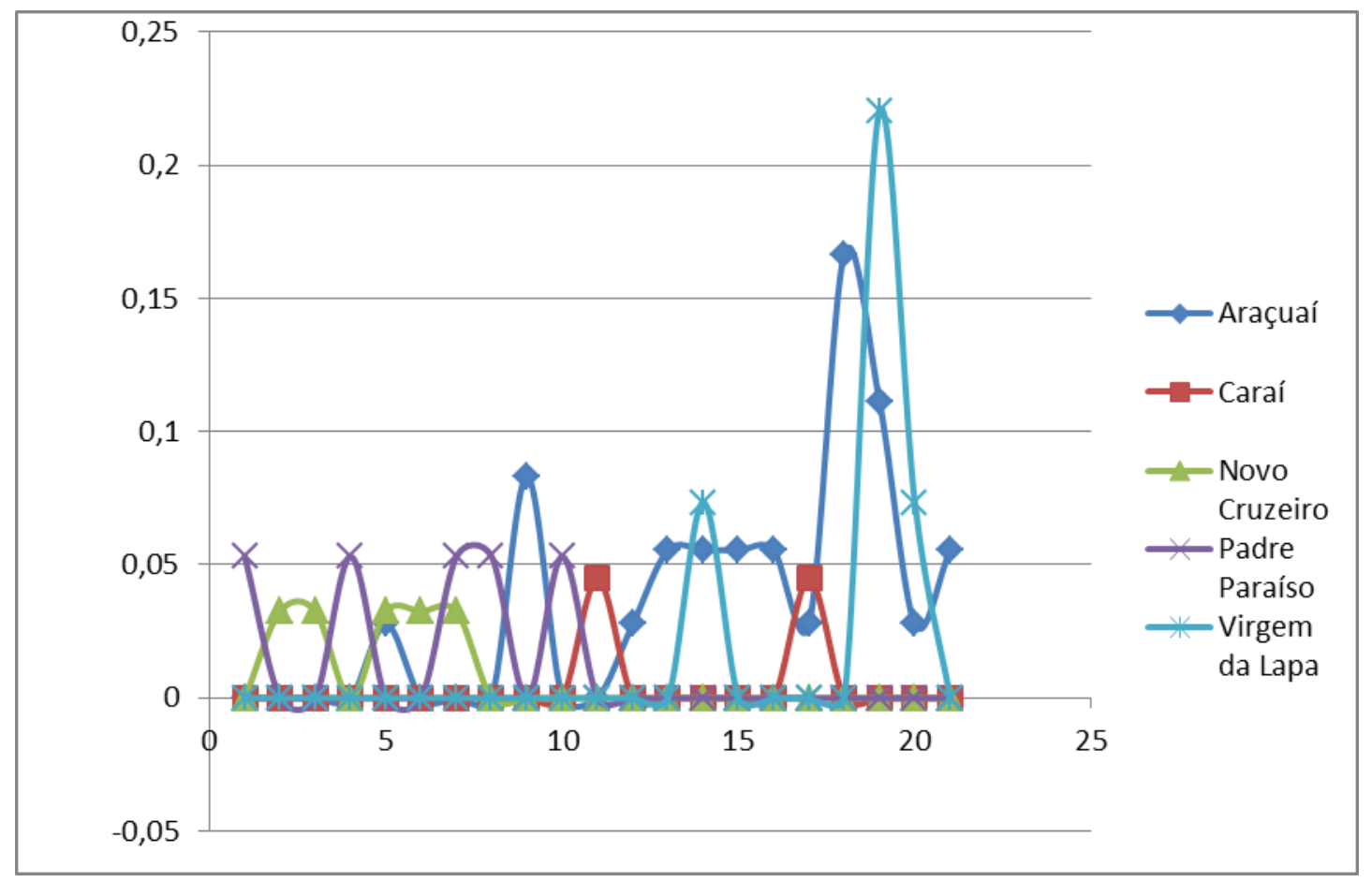

Fonte: SIH/SUS; IBGE (2015)

A distribuição da média de internação por transtorno mental devido ao uso de álcool, em relação à população de cada município, mostrou que embora as 
internações em Araçuaí tivessem maior quantidade, a média maior, foi no município de Virgem da Lapa, média de internações de 0,36 por 1000 habitantes.

Nos municípios de Araçuaí, Caraí, Novo Cruzeiro, Padre Paraíso e Virgem da Lapa apresentou crescimento no ano de 2014, sendo os meses de Agosto, Setembro e Outubro com as maiores taxas.

Quanto à idade, as variáveis da pesquisa apontaram duas faixas etárias. $\mathrm{Na}$ faixa etária 1 estão compreendidas as idades de: entre menor de 1 ano, 20 a 29 anos, 30 a 39 anos, 40 a 49 anos, 50 a 59 anos, 60 a 69 anos, 70 a 79 anos.

As idades entre 30 a 49 anos tem maior ocorrência de internações num total de 17 internações, seguido das idades 40 a 49 anos como mostra o gráfico 3 .

GRÁFICO 3 - Internação por faixa etária 1 segundo o município por transtorno mental devido ao uso de álcool.

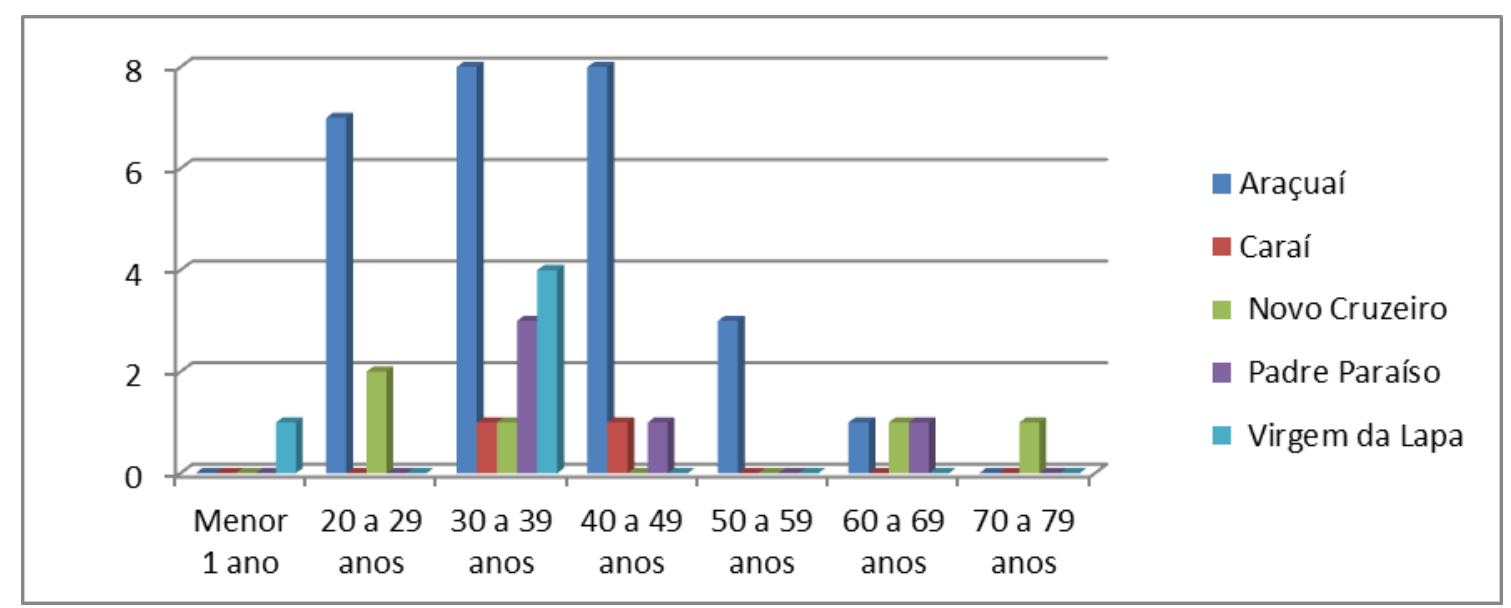

Fonte: SIH/SUS (2015)

GRÁFICO 4 - Média de internação por faixa etária 1 segundo o município por transtorno mental devido ao uso de álcool. 
Revista Multidisciplinar do Nordeste Mineiro, v1. 2020/01 ISSN 2178-6925

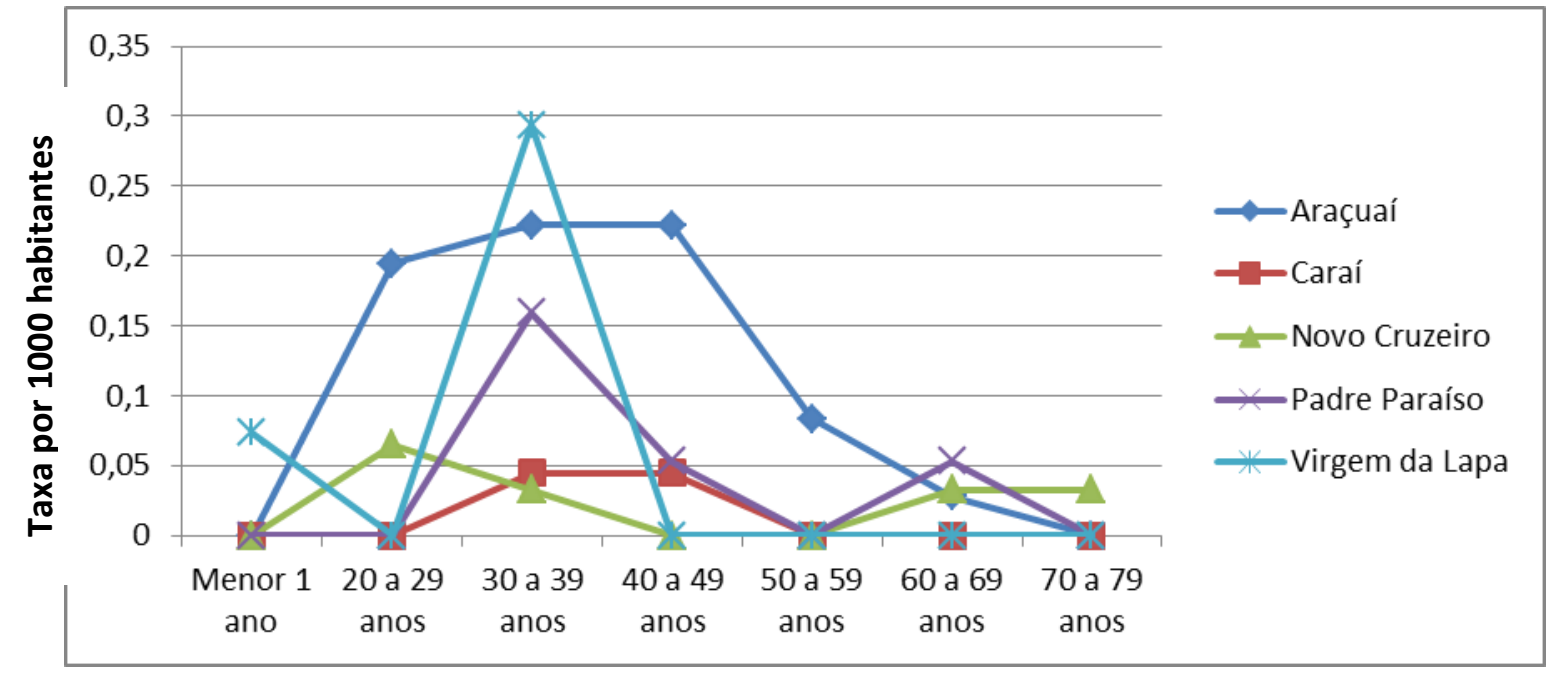

Fonte: SIH/SUS; IBGE (2015)

Percebe-se que em Virgem da Lapa a idade de 30 a 39 anos houve um crescimento (GRÁF. 4).

Na faixa etária 2 estão compreendidas as idades de: menor de 1 ano, 20 a 24 anos, 25 a 29 anos, 30 a 34 anos, 35 a 39 anos, 40 a 44 anos, 45 a 49 anos, 50 a 54 anos, 60 a 64 anos, 65 a 69 anos, 75 a 79 anos (GRAF. 5).

GRÁFICO 5- Internação por faixa etária 2 segundo o município por transtorno mental devido ao uso de álcool por dados de população

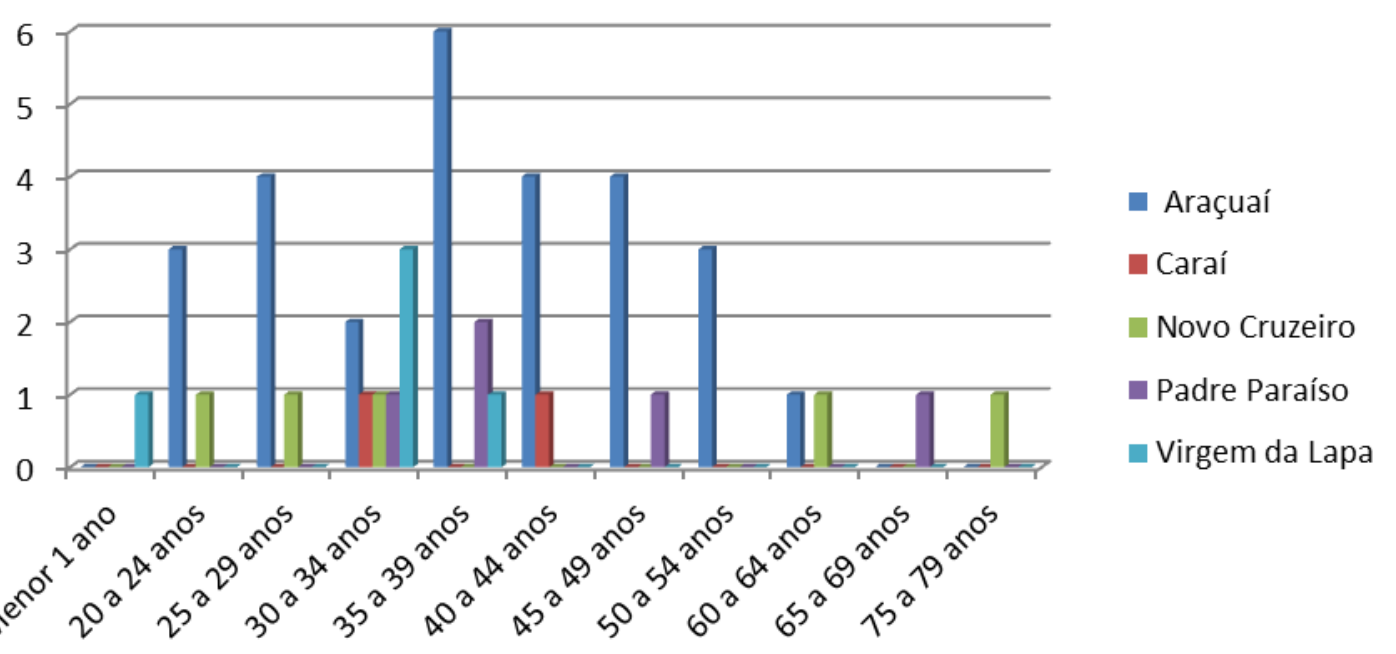


Revista Multidisciplinar do Nordeste Mineiro, v1. 2020/01 ISSN 2178-6925

Fonte: SIH/SUS; IBGE (2015)

Percebe-se que a idade de 35 a 39 anos tem maior prevalência de internação sendo 9 casos. Nesta mesma idade Araçuaí é o munícipio com maior quantidade (TAB. 5 e GRÁF. 5)

A idade de 30 a 34 anos teve ocorrência de internações em todos os municípios (TAB. 5).

Em relação à média de internações o município de Virgem da Lapa teve maior prevalência de internação (GRÁF. 6).

GRÁFICO 6- Média de internação por faixa etária 2 segundo o município por transtorno mental devido ao uso de álcool por dados de população

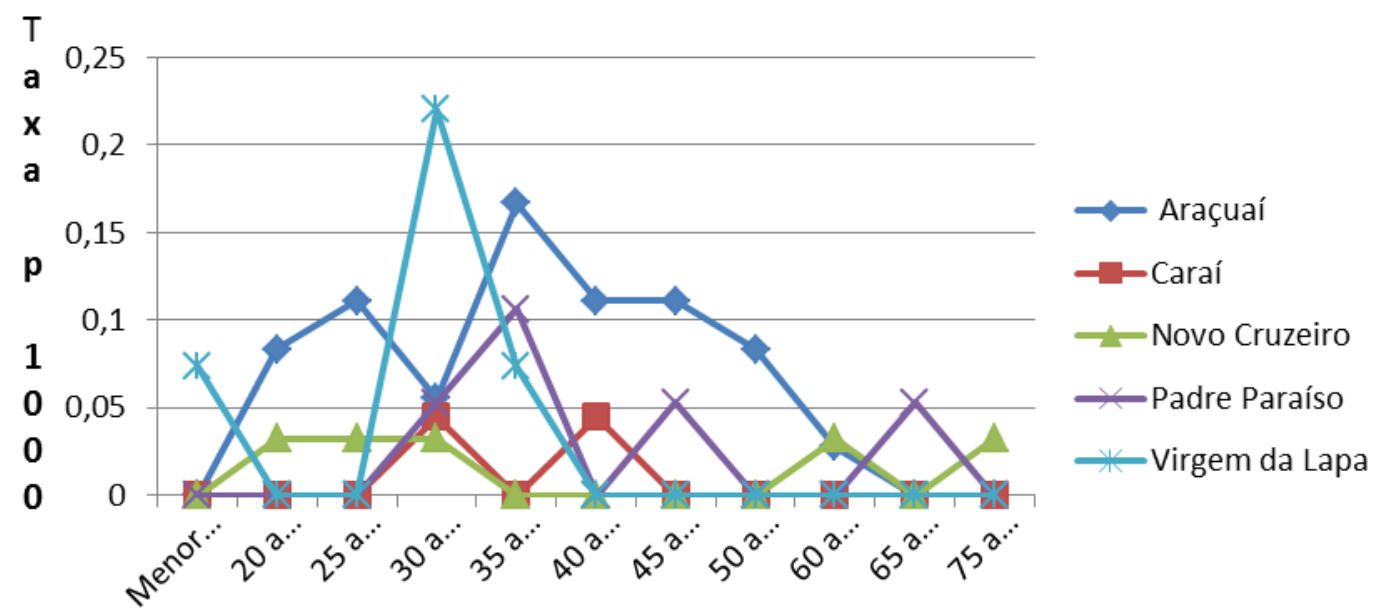

Fonte: SIH/SUS; IBGE (2015)

Em relação à cor e raça segundo pesquisa, no período discriminado, a cor parda apresenta maior prevalência de internações e o município de Araçuaí teve maior ocorrência de internações pela cor parda (GRÁF. 7).

GRÁFICO 7- Internação por cor/raça segundo o município por transtorno mental devido ao uso de álcool. 
Revista Multidisciplinar do Nordeste Mineiro, v1. 2020/01 ISSN 2178-6925

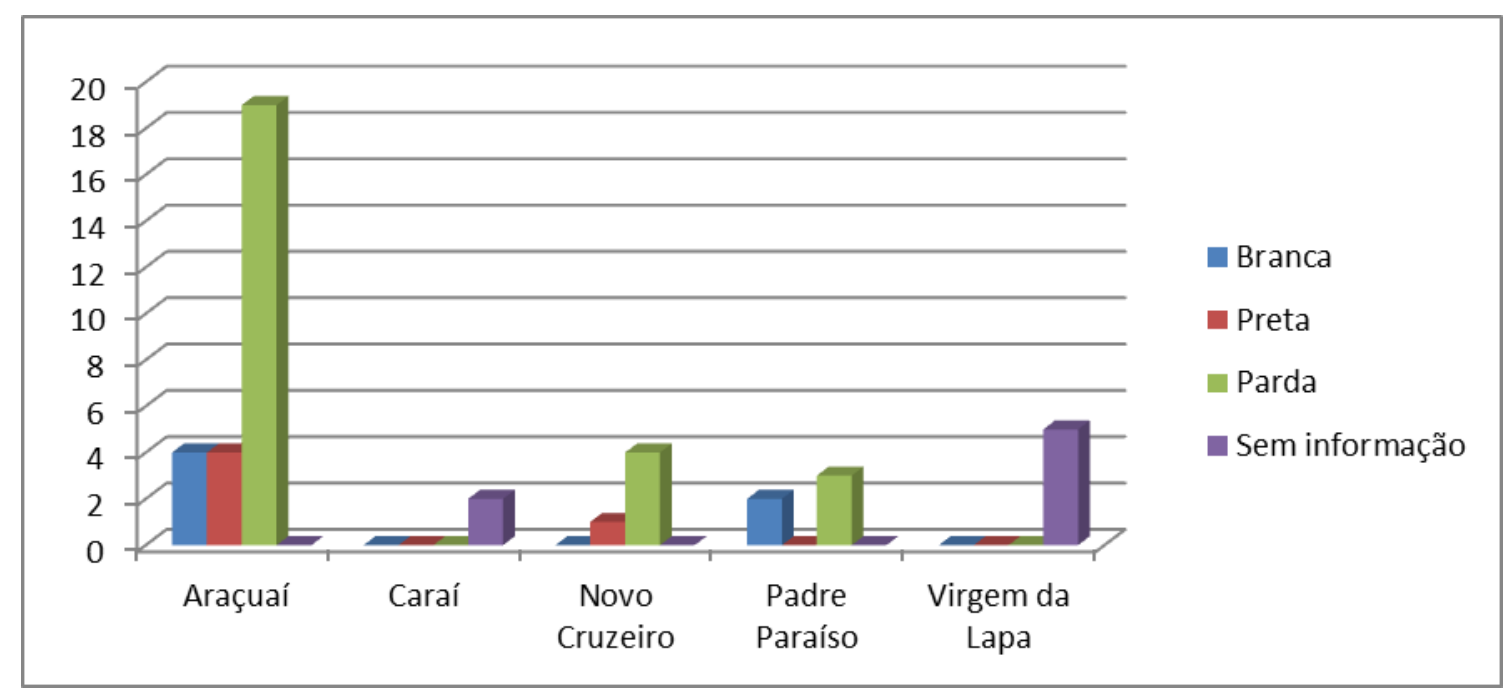

Fonte: SIH/SUS (2015)

GRÁFICO 8- Internação por sexo segundo o município por transtorno mental devido ao uso de álcool

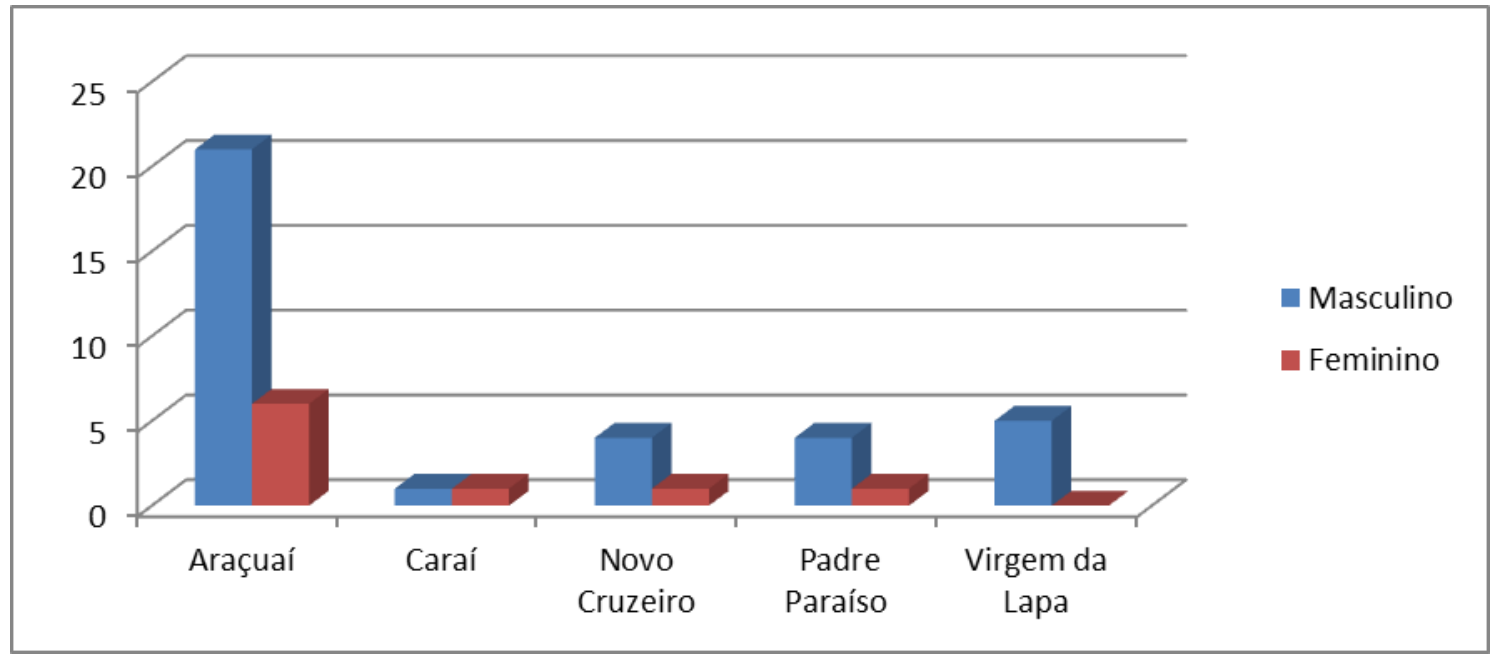

Fonte: SIH/SUS (2015)

O sexo masculino apresenta maior prevalência de internações por transtorno mental devido ao uso de álcool (GRÁF. 8).

\section{Discussão}


Pode-se dizer que a realização deste trabalho, bem como os aspectos abordados dentro da temática de transtornos mentais devido ao uso de álcool levanos a buscar meios de compreender como o uso de álcool está se tornando um problema para a população em questão.

Inicialmente, cabe destacar a importância para os lançamentos no banco de dados do SIH/SUS, principalmente na variável de Transtorno mentais devido ao uso de álcool, para o conhecimento da realidade do uso de álcool que é crescente e consumido por jovens e adultos.

Neste trabalho, os dados coletados no Sistema de Informação Hospitalar do Sistema Único de Saúde (SIH/SUS) no período de 2008 a 2014 mostrou um número baixo em relação à população dos municípios de Araçuaí, Caraí, Coronel Murta, Itinga, Novo Cruzeiro, Padre Paraíso, Ponto dos Volantes e Virgem da Lapa (Microrregião de Araçuaí, Vale Jequitinhonha, Minas Gerais, Brasil).

Constata-se com os resultados apresentados, que pode ser apontadas quatro hipóteses. A primeira hipótese seria que munícipios com maior população tem maior quantidade de internações, logo, o município de Araçuaí, com maior população, apresentou média total de $2,45 \%$ de internações por transtorno mental devido ao uso de álcool, seguido dos municípios de Novo Cruzeiro, Padre Paraíso e Virgem da Lapa com 0,45\%, e por ultimo, com 0,18\%, o município de Caraí. Entretanto, em relação à média de internações/ população do município, o município de Virgem da Lapa, quinto município em numerosidade populacional da Microrregião de Araçuaí, apresentou maior média no mês de setembro do ano de 2014 , sendo $0,22 \%$, sendo a maior taxa.

A segunda hipótese a ser apontada é que a cada ano, os casos aumentam, assim, dentre o período pesquisado houve um crescimento de internações. O consumo de álcool e o nível socioeconômico tem relação, sendo o Vale Jequitinhonha, conhecido como vale da pobreza e da miséria, apontamos que a microrregião de Araçuaí tem um alto índice do problema de transtorno mental devido ao uso de álcool. Existe uma relação entre pobreza e saúde mental, pois as condições da pobreza como o desemprego, baixo nível de instrução, privação e desabrigo, é um dos mecanismos causal para os transtornos por uso de substâncias em pobres (OMS, 2001, p.13).

A terceira hipótese levantada é que a idade entre 30 a 40 anos tem maior ocorrência de internações, portanto, quanto às faixas etárias, idades entre 30 a 39 
anos, tiveram as maiores taxas de internação, idade que é de produtividade de trabalho, podendo indicar problemas de desemprego na região, pois, quando um indivíduo está em situação de desemprego, apresenta várias reações com o problema desde a mudança de humor e insônia até uma adaptação patológica, o que classifica a terceira fase, sendo o alcoolismo (PINHEIRO, L.R.S.; MONTEIRO, J.K., 2007, p. 40).

A quarta hipótese era que o sexo masculino tem mais ocorrência de internações por transtorno mental devido ao uso de álcool, logo, os dados da pesquisa revelaram que o sexo masculino teve maior prevalência de internações, $7 \%$ e mulheres $1,8 \%$, o que corrobora com os achados na literatura citado por Andrade e Wang (2008), "nos homens, o impacto dos transtornos mentais por uso de álcool e drogas é seis vezes maior do que nas mulheres, compreendendo $25 \%$ da carga total por transtornos psiquiátricos" (ANDRADE, L.H.S.; WANG, Y.P., 2008, p.66).

A pesquisa ainda aponta, que taxas maiores de internações de pacientes foram na cor parda, em contraponto, estudos transversais evidenciassem que os transtornos mentais e comportamentais (TMC) são mais frequentes entre mulheres, negros, pobres, desempregados, tabagistas, doentes crônicos, indivíduos com baixo apoio social ou que sofreram eventos estressantes, indivíduos com baixa escolaridade ou cujas mães tinham baixa escolaridade (ANSELMI, L. et al, 2008, p. 27).

Segundo Machado, M.C.P. (2012) no ano de 2004, 10\% a 12\% da população mundial era dependente de alcoólicos, no Brasil (dados de 2008) 12,3\% da população podia ser considerada dependente de álcool, sendo que a prevalência é de $17,1 \%$ para os homens e 5,7\% para as mulheres, apontando que o alcoolismo crônico é o $3^{\circ}$ fator de afastamento de mais de 15 dias no nosso país levando a benefícios como auxílio doença e aposentadoria por invalidez.

$\mathrm{Na}$ análise dos resultados, o período de 2008 a 2014, apresentou um número baixo de internações por transtorno mental devido ao uso de álcool, 44 casos. Outro dado a ser questionado é que do ano de 2008 ao primeiro semestre de 2009, não houve internações, podendo ser apontando a ausência de lançamentos no sistema. Surgindo assim, a hipótese questões de faturamento, onde o lançamento pode ter sido com outro diagnóstico, como a desnutrição, por exemplo. 
As variáveis pesquisadas são importantes, pois, "sabe-se que características como gênero, idade, grau de instrução e estado civil pode influenciar no uso nocivo e desenvolvimento da dependência do álcool" (SILVA, C.R. et al, 2009, p. 103).

Na microrregião de Araçuaí, o município de Araçuaí possui CAPS AD, mostrando que é relevante a necessidade de prevenção ao uso de álcool na região, pois em pesquisa realizada em cinco regionais do Estado de São Paulo apontou que internação devido ao uso de álcool e drogas foram em Campinas = 1,0; Piracicaba =0,2; São José do Rio Preto = 0,2; Mogi = 24,0; São José dos Campos $=0,2$, os autores apontam para um decréscimo nessas internações, supondo que seria devido a atuação do CAPS AD na região (MORAIS, M.L.S; et al, 2009, p. 119).

Para OMS (2001) "existem valores culturais e religiosos associados com baixos níveis de uso do álcool”, uma estratégia a ser utilizada é que grupos religiosos e comunitários tenha o compromisso de trabalhar com a prevenção do uso do álcool para evitar os transtornos mentais devido ao uso do álcool. Pois:

\begin{abstract}
A intervenção precoce em face de tais problemas, as intervenções psicológicas, o tratamento dos efeitos nocivos do álcool (inclusive sintomas de abstinência e outras consequências médicas), o ensino de novas aptidões para fazer face a situações associadas com alto risco de consumir bebida e sofrer recorrência, a educação da família e a reabilitação são as principais estratégias de efetividade comprovada para o tratamento de problemas relacionados com a dependência de álcool e outras substâncias (OMS, 2001, p.67).
\end{abstract}

Além do tratamento ambulatorial e a internação dos dependentes de álcool, as técnicas psicológicas tem grande eficácia. Neste panorama, a atuação da psicologia é necessária, junto com políticas públicas na microrregião de Araçuaí para que foquem na prevenção do uso de álcool, pois o uso da substância inicia muitas vezes na adolescência, sendo assim, um trabalho com as escolas e com toda a Rede de Garantia dos Direitos da Criança e do Adolescente, como CRAS, CREAS, Conselho Tutelar, Ministério Público, entre outros, atuarem com projetos que previnam o uso das bebidas etílicas como formas de reduzir o consumo do álcool.

O uso da abordagem cognitivo comportamental, com entrevistas 
Alcoólicos Anônimos, durante e depois do tratamento profissional, são associados com melhores resultados do que o tratamento medicamentoso somente, sendo necessária, o acompanhamento psicológico do grupo familiar para iniciação e manutenção do tratamento (OMS, 2001, p.67).

\section{Conclusão}

Certamente, o álcool é um assunto que repercute nos âmagos da sociedade, pois ele é um dos fatores responsáveis pela desestruturação das famílias, causando muitas vezes, rompimento nos laços afetivos.

No ambiente familiar que tem o alcoolista, sendo este portador de transtorno mental devido ao uso de álcool, é um lar de adoecimento, com instabilidade, insegurança e conflituoso, o que também influencia na saúde mental dos demais membros, pois estes podem desenvolver transtornos mentais, como a depressão, por exemplo.

A iniciativa desta pesquisa é fazer um levantamento epidemiológico de internações de transtornos mentais causados pelo uso de álcool na microrregião de Araçuaí, situada no Vale Jequitinhonha e assim mostrou que o consumo do álcool na região é preocupante, sendo necessário que a população possa ter conhecimento dos malefícios do seu consumo e que é necessário que possíveis políticas públicas possam intervir na prevenção dos transtornos mentais devido ao uso de álcool.

O uso abusivo de bebidas etílicas podem favorecer a intercorrências no ambiente familiar como a violência intrafamiliar por exemplo.

Portanto, viabilizar pesquisas nesta região quanto ao uso de álcool apontará estatisticamente uma visão da problemática na relação entre álcool e saúde mental. Neste sentido, há necessidade em desenvolver pesquisas de campo, utilizando questionários e escalas, pois estas pesquisas favorecerão para apontar os fatores epidemiológicos, prevalência e incidência do uso de álcool e desencadeamento do transtorno mental quanto ao uso de álcool, no Vale Jequitinhonha, microrregião de Araçuaí. Todavia, a psicologia através das suas áreas de atuação em saúde mental em interface com as políticas públicas, é ferramenta para uma interlocução entre a saúde mental e o uso de álcool na região. 
O campo da psicologia, em suas teorias associados ao tratamento profissional, em parceria com o grupo dos Alcoólicos Anônimos, durante e depois do tratamento profissional, são associados com melhores resultados do que o tratamento medicamentoso somente, sendo necessário, o acompanhamento psicológico do grupo familiar para iniciação e manutenção do tratamento.

\section{Referências}

ANDRADE, L.H.S.; WANG, Y.P. Epidemiologia dos transtornos psiquiátricos. In ALVARENGA, P.G.; ANDRADE, A.G. FUNDAMENTOS EM PSIQUIATRIA. 1ªed. Barueri: Manole, 2008, Cáp. 4, p. 65-88.

ANSELMI, L. et al Prevalência e determinantes precoces dos transtornos mentais comuns na coorte de nascimentos de 1982, Pelotas, RS. Rev. Saúde Pública. 42 (Supl.2), p. 26-33, 2008.

BRASIL. Instituto Brasileiro de Geografia e Estatística - IBGE (Censo Demográfico, IBGE Cidades). . Ministério da Saúde (DATASUS), 2015.

$$
\text { Disponível }
$$

em

http://memoria.ebc.com.br/agenciabrasil/noticia/2013-05-20/oms-doencas-mentaise-neurologicas-atingem-cerca-de-700-milhoes-de-pessoas-alerta-oms > Acesso em 21/10/2014 às 18:12.

Secretaria Nacional de Políticas sobre álcool e drogas. Prevencão dos problemas relacionados ao uso de drogas: capacitacão para conselheiros e lideranças comunitárias. Ministério da Justiça, 6믈 ed. Brasília, DF: SENAD-MJ/NUTE-UFSC, 2014, $312 \mathrm{p}$.

CID-10: classificação de transtornos mentais e de comportamento da CID-10: descrições clínicas e diretrizes diagnósticas. Porto Alegre : Artes Médicas, 1993.

DSM-IV - Manual diagnóstico e estatístico de transtornos mentais. Porto Alegre, Ed. Artes Médicas, 1995. 
MACHADO, Maria Cristina Palhares. TRANSTORNOS MENTAIS: DADOS . ESTATíSTICOS. 26ª Jornada da Amint. NOVEMBRO/2012.

MACHADO, R.M, COSTA JUNIOR, M.L.; Alcoolismo na região centro oeste de Minas Gerais: perfil sócio demográfico, clínico e distribuição geográfica. Revista Eletrônica Saúde Mental, Álcool e Drogas. №8, p. 71-78, Maio-Agosto/2012.

MORAIS, M.L.S. Um estudo avaliativo das ações de saúde mental no estado de São Paulo. Saúde em Debate, Rio de Janeiro, v. 33, n. 81, p. 112-128, jan./abr. 2009.

Organização Mundial de Saúde, Organização Pan-Americana de Saúde. Relatório sobre a Saúde no mundo2001: Saúde mental: nova concepção, nova esperança. Geneva: OMS, 2001.

$$
\text { 2014. Disponível em }
$$

<http://translate.googleusercontent.com/translate c?depth=1\&hl=pt-

R\&prev=/search\%3Fq\%3DWHO\%26client\%3Dfirefox-

a\%26hs\%3DVxM\%26rls\%3Dorg.mozilla:pt-

BR:official\%26channel\%3Dsb\%26biw\%3D1366\%26bih\%3D631\&rurl=translate.goo gle.com.br\&sl=en\&u=http://www. who.int/mediacentre/factsheets/fs220/en/\&usg=AL kJrhggAftRoDK4xWcGuiOtUJe5Pb6k1g > Acesso em 28/09/2014 as 17:43.

PINHEIRO, L.R.S.; MONTEIRO, J.K. Reflitindo sobre o desemprego e agravos à saúde mental. Cadernos de Psicologia Social do Trabalho. 2007, Vol. 10, n. 2, pp. 35-45.

UNIVERSIDADE FEDERAL DE MINAS GERAIS. Disponível em < https://www2.ufmg.br/polojequitinhonha/O-Vale/Sobre-o-Vale> Acesso em $13 / 08 / 2015$

SILVA, C. R., et al, Comorbidade psiquiátrica em dependentes de cocaína/crack e alcoolistas: um estudo exploratório. Aletheia, 30, p. 101-112, jul/dez 2009.

SALOMAO, P. E. A. et al. As Tecnologias de Informação e Comunicação (TIC) no ensino superior. Revista Multidisciplinar do Nordeste Mineiro, v. 1, 2018. 
Revista Multidisciplinar do Nordeste Mineiro, v1. 2020/01 ISSN 2178-6925

SILVA, D. F. Transtornos mentais e pobreza no Brasil: uma revisão sistemática / Dilma Ferreira da Silva. Recife: D. F. da Silva, 2010. 